\title{
Construction of transcriptional regulatory networks based on found transcription factors and their binding sites in annotated bacterial genomes
}

\author{
Lakhova T.N. ${ }^{1 *}$, Oshchepkov D.Yu. ${ }^{1}$, Mukhin A.M. ${ }^{1}$, Lashin S.A. ${ }^{1,2}$ \\ ${ }^{1}$ Kurchatov Genomic Center of the Institute of Cytology and Genetics, SB RAS, Novosibirsk, Russia \\ ${ }^{2}$ Novosibirsk State University, Novosibirsk, Russia \\ *tlakhova@bionet.nsc.ru
}

Key words: transcriptional regulatory networks, transcription factors, Cytoscape

Motivation and Aim: The regulation of gene expression is important in bacterial life, since for most genes it can change dramatically in response to environmental variations. In recent years, a huge amount of whole-genome sequencing data has been accumulated. The potential of these data in terms of analyzing the regulation of gene expression is far from being fully disclosed. Moreover, the amount of data relevant to the study of expression increases every year. Despite the successes achieved in the study of regulatory interactions in bacteria, there are still many gaps in this field. For example, for the most studied model organism, Escherichia coli, it has been estimated that only portion between $10 \%$ and $30 \%$ of the real regulatory interactions are well studied by now [1]. There is even less information available for other bacterial organisms. Therefore, the construction of transcriptional regulatory networks (TRNs) based on genomic information is an essential task of modern systems biology. The study presents an algorithm for constructing such TRNs for bacterial genomes and its software implementation.

Methods and Algorithms: We used data on transcription factors and their binding sites. These data were obtained using the algorithm and pipeline we developed earlier [2]. The pipeline includes external programs: Operon-mapper, BoBro2.0, Tomtom from MEME Suite, as well as our own script written in Python using the pandas, re and Biopython libraries. The regulatory networks were constructed using Python and the pandas. Cytoscape was used to visualize TRNs.

Results: We reconstructed several TRNs of bacterial genomes from the database of the Kurchatov Genomic Centre of ICG SB RAS. The functionality of the program allows one to choose to build a network with or without an operon structure.

Conclusion: The obtained TRNs can be used both for further structural analysis and for creating mathematical models on their base.

Acknowledgements: The study is supported by the Kurchatov Genomic Centre of the Institute of Cytology and Genetics, SB RAS (075-15-2019-1662).

\section{References}

1. Santos-Zavaleta A. et al. RegulonDB v 10.5: tackling challenges to unify classic and high throughput knowledge of gene regulation in E. coli K-12. Nucleic Acids Res. 2019;47(D1):D212D220.

2. Lakhova T.N. et al. Development of algorithms for finding transcription factors and their binding sites in annotated bacterial genomes. Systems Biol. Bioinformatics. 2020;87. (in Russian) 